\title{
Mapping the mechanical microenvironment in the ovary
}

Thomas I. R. Hopkins ${ }^{1,2}$, Victoria L. Bemmer ${ }^{1}$, Stephen Franks ${ }^{2}$, Carina Dunlop ${ }^{3}$, Kate Hardy ${ }^{2}$, lain E. Dunlop ${ }^{1 *}$.

1 Department of Materials, Imperial College London, Exhibition Road, London SW7 2AZ, U.K.

2 Institute of Reproductive and Developmental Biology, Imperial College London, Hammersmith Campus, London, W12 0NN, U.K.

3 Department of Mathematics, University of Surrey, GU2 7XH, U.K.

\section{${ }^{*}$ Corresponding author: lain E. Dunlop (i.dunlop@imperial.ac.uk)}

\begin{abstract}
Follicle development in the human ovary must be tightly regulated to ensure cyclical release of oocytes (ovulation), and disruption of this process is a common cause of infertility. Recent ex vivo studies suggest that follicle growth may be mechanically regulated, however the actual mechanical properties of the follicle microenvironment have remained unknown. Here we map and quantify the mechanical microenvironment in mouse ovaries using colloidal probe atomic force microscope (AFM) indentation, finding an overall mean Young's Modulus $3.3 \pm 2.5 \mathrm{kPa}$. Spatially, stiffness is low at the ovarian edge and centre, which are dominated by extra-follicular ECM, and highest in an intermediate zone dominated by large follicles. This suggests that large follicles should be considered as mechanically dominant structures in the ovary, in contrast to previous expectations. Our results provide a new, physiologically accurate framework for investigating how mechanics impacts follicle development and will underpin future tissue engineering of the ovary.
\end{abstract}

Keywords: Ovary, AFM Indentation, Tissue Stiffness

\section{Introduction}

The ovary must precisely regulate the activation and development of follicles to release, in humans, exactly one oocyte (egg) per cycle. In contrast follicle dysregulation leads to disease and compromised fertility. In particular in polycystic ovary syndrome (PCOS), follicle development is impaired with consequent reduction in the rate of ovulation $(1,2)$, while in the other direction uncontrolled and accelerated follicle development depletes follicle stocks leading to premature ovarian insufficiency (POI). In recent years it has been proposed that the mechanical properties of the ovarian environment are key to regulating follicle growth, in combination with previously identified factors of pituitary hormones, steroid hormones and growth factors (3-7). There is increasing 
evidence that the follicle responds to its mechanical environment, which either suppresses or enhances growth, with this mechanical environment controlled in turn by hormones or growth factors.

Evidence for mechanical regulation in follicle development includes on a fundamental cellular level the known role of the mechanosensitive Hippo $(8,9)$ and Akt $(10-13)$ signalling pathways. The most compelling evidence for mechanical control of follicle development, however, comes from experiments that have cultured follicles in vitro in biomaterial gels of controlled stiffness(14-19). These studies have shown that varying the gel stiffness can control follicle properties including growth, steroidogenesis and antrum formation, with a postulated switch from suppressive to a permissive environment for follicle development when the microenvironment stiffness drops below a threshold value in the range range $0.1-1 \mathrm{kPa}$ (Young's Modulus) $(14,15,19)$. These experiments, combined with the knowledge that ECM content varies spatially through the ovary (20-23), have led to the hypothesis that follicle development is mechanically regulated by the different mechanical microenvironments of different ovarian regions (15,24-26). Since extra-follicular ECM density also varies dynamically across the menstrual cycle such ECM-driven mechanical control is a plausible means of dynamically regulating follicle development (27-30).

Despite these exciting concepts derived from in vitro culture, the actual mechanical properties of the follicle microenvironment, and how these vary spatially within the ovary remain unknown. Indeed the only substantial mechanical characterization of the ovary to date is a recent study that measures the bulk stiffness of the whole ovary using external indentation (31). Biomechanical control of follicle development in the actual ovary hence remains hypothetical. Here, we map the mechanical properties of the mouse ovarian interior with high spatial resolution, directly revealing the mechanical conditions that follicles will experience in the ovarian microenvironment. We use colloidal probe atomic force microscopy (AFM) indentation (32-36) to generate a spatial profile of stiffness (Young's modulus) across a bisected ovary. We find a mean ovarian stiffness of $3.3 \pm 2.5 \mathrm{kPa}$, with local fluctuations attributable to microstructural variation, but importantly also with substantial structural variation across the ovary. In contrast to prior expectations, the ovary is relatively soft at the edge and centre, regions that are dominated by extra-follicular ECM rich in collagen IV. However, in an 
intermediate zone between the centre and edge, where the tissue is dominated by large follicles, the ovary is much stiffer. This indicates that large follicles themselves are the dominant mechanical objects in this system, and focuses attention on direct follicle-follicle interactions as a likely key factor in follicle mechanoregulation.

\section{Results}

We measured the local stiffness (characterized as Young's modulus, E), using colloidal probe indentation with the AFM (for a full description see materials and methods). Briefly, a mouse ovary (25-29 days post-partum) was collected and embedded in an agar gel. The ovary and gel were then bisected with a sharp microtome blade to reveal the interior surface of the ovary (Fig 6). Using AFM, the cut surface of the ovary was probed at intervals from edge to edge across the ovary, using a spherical colloidal probe $(10.8 \mu \mathrm{m}$ diameter $)$ mounted on the AFM cantilever. Colloidal probe indentation was selected to generate a reliable mechanical measurement since the Young's modulus value will be averaged over an area of the same order of the sphere diameter, avoiding highlylocalized effects. Equally, the $\sim 10 \mu \mathrm{m}$ probe size is much less than the $\sim 2 \mathrm{~mm}$ size of the ovary itself, enabling a useful mapping of variation within the ovarian interior. At each site, a forceindentation curve was measured, and the Young's modulus was extracted via a fit to the Hertz model (see Materials and Methods) (37). The fit was made to data at moderately high indentation, again to generate a reliable value for by averaging over a sufficiently large tissue volume, and to avoid any artefacts due to possible surface damage during ovary bisection.

Young's modulus was mapped in a line-section across the ovary, illustrated in Figure 1D. The linesection was constructed by selecting locations spaced $50-100 \mu \mathrm{m}$ apart across the ovarian interior surface and passing through its centre (insofar as this could be achieved by eye). At each location, a map of Young's modulus was created $30 \times 30 \mu \mathrm{m}(6 \times 6$ grid of indentations with $5 \mu \mathrm{m}$ spacing). This procedure reflected the construction of the AFM, which combined a $30 \times 30 \mu \mathrm{m}$ local piezo drive, and a micrometer-driven sample stage for larger spacing. To demonstrate reliability and technical reproducibility, the line-section was constructed to incorporate indentation at several locations on the stiffer agar gel surrounding the ovary, at both the start and the end of the section. 
bioRxiv preprint doi: https://doi.org/10.1101/2021.01.03.425098; this version posted January 4,2021 . The copyright holder for this preprint (which was not certified by peer review) is the author/funder, who has granted bioRxiv a license to display the preprint in perpetuity. It is made available under aCC-BY-NC-ND 4.0 International license.

The stiffness of agar was consistently of order $15 \mathrm{kPa}$, and most importantly showed no appreciable alteration between the start and end of the line-sections (Figure 4A). To confirm repeatability and that indentation does not significantly damage the tissue surface, we constructed multiple maps at the same location, showing no significant difference in the values recorded in first and second indentation $(p>0.05$, Supplementary Figure 1). 

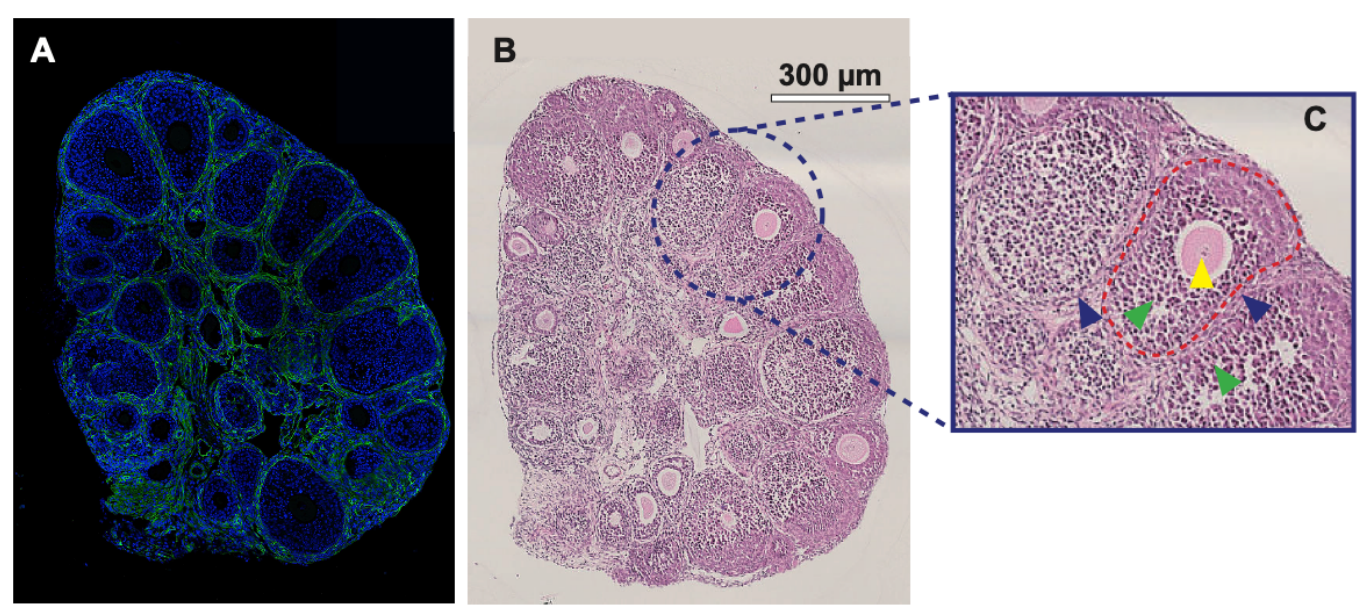

D

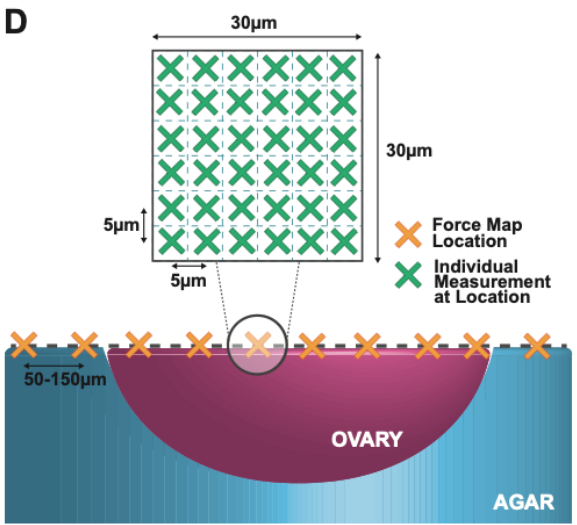

E

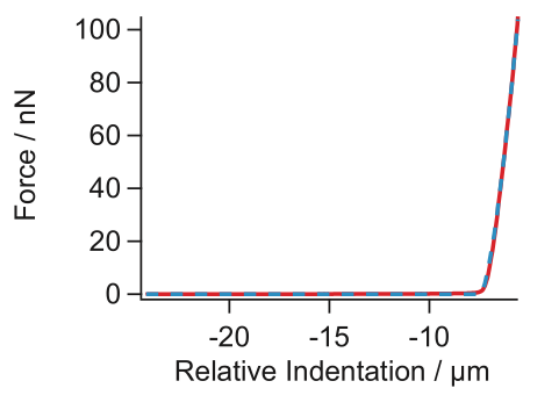

Figure 1. Measuring the mechanical properties of the ovarian microenvironment. $(A, B)$ Representative histology images showing the exposed surface of a bisected ovary, on which the mechanical indentation measurements were performed. Staining is (A) Immunofluorescence showing collagen IV as a marker for ECM (green) and DAPI staining of cell nuclei (blue). (C) Closeup showing a follicle (outer border given by red dotted line), indicating the oocyte (indicated by yellow triangle), granulosa cells (green triangle), theca cell layer (blue triangle). D) Schematic of indentation line-scan: a series of locations across the ovary interior surface $(X)$ with spacing $50-100 \mu \mathrm{m}$ are selected. At each location, a $30 \times 30 \mu \mathrm{m}$ map is created by indenting at a $6 \times 6$ grid of sites (solid dots) with $5 \mu \mathrm{m}$ spacing. (E) A representative curve of force, $F, v s$ indentation $\delta$ (the values on the $x$-axis are relative since the surface location $\delta_{0}$ is unknown until after fitting) curve, showing a fit to the Hertz model using data at moderate indentation as per the methods section. 


\section{The ovary is a fairly soft tissue but with very broad micromechanical variation}

We first considered the overall distribution of the Young's modulus values measured ( $n=4$ ovaries,

Fig. 2, Left Panel). It can be seen that a substantial range of values (c. $0.5 \mathrm{kPa}$ to $10 \mathrm{kPa}$.) is measured, summarized by a mean value of $3.3 \pm 2.5 \mathrm{kPa}$ or median $2.6 \pm 2.8 \mathrm{kPa}$ (Fig 2 , Right Panel) (where \pm signifies standard deviation and interquartile range respectively). Interestingly, the distribution is heavily skewed towards low stiffnesses, with a large number of values below $1 \mathrm{kPa}$. Comparing the mean and standard deviation values we have measured for the ovarian stiffness with those measured for other tissues $(32,33,38-41)$, (Figure 3), we see that the ovary emerges as an overall fairly soft tissue, comparable with the kidney or fat, but not as soft as e.g. the brain. These overall values are in good accordance with recent measurements of whole-ovary stiffness using exterior indentation, which quoted c. $2 \mathrm{kPa}$ for 'reproductively young' mouse ovaries that are roughly comparable with those measured here. However looking in more detail reveals a more complex picture (31). The ovarian microenvironment is seen to range from very low stiffnesses usually associated with the brain as the softest tissue up to values that approach those associated with stiff tissues such as muscle. Hence it becomes clear that mechanical variations within the ovarian microenvironment may be as significant as variations between the ovary and other tissues. As a further contextualization, we compare our measured values for the ovarian stiffness with the permissive stiffnesses for follicle activation in controlled-stiffness gels discussed above (1419,42,43). In this work, examples of Young's modulus values considered permissive for follicle development are in the range $100-800 \mathrm{~Pa}$ (Supplementary Table 1) $(14,15,19)$, however the upper limit is not necessarily precisely defined. Given that the ovary does indeed exhibit a substantial number of sites where stiffness is at sub-kPa values, it is possible that the requirements for 'permissiveness' could be fulfilled. 


\section{Distribution of Young's Modulus \\ (all sites, $\mathrm{n}=4$ ovaries)}
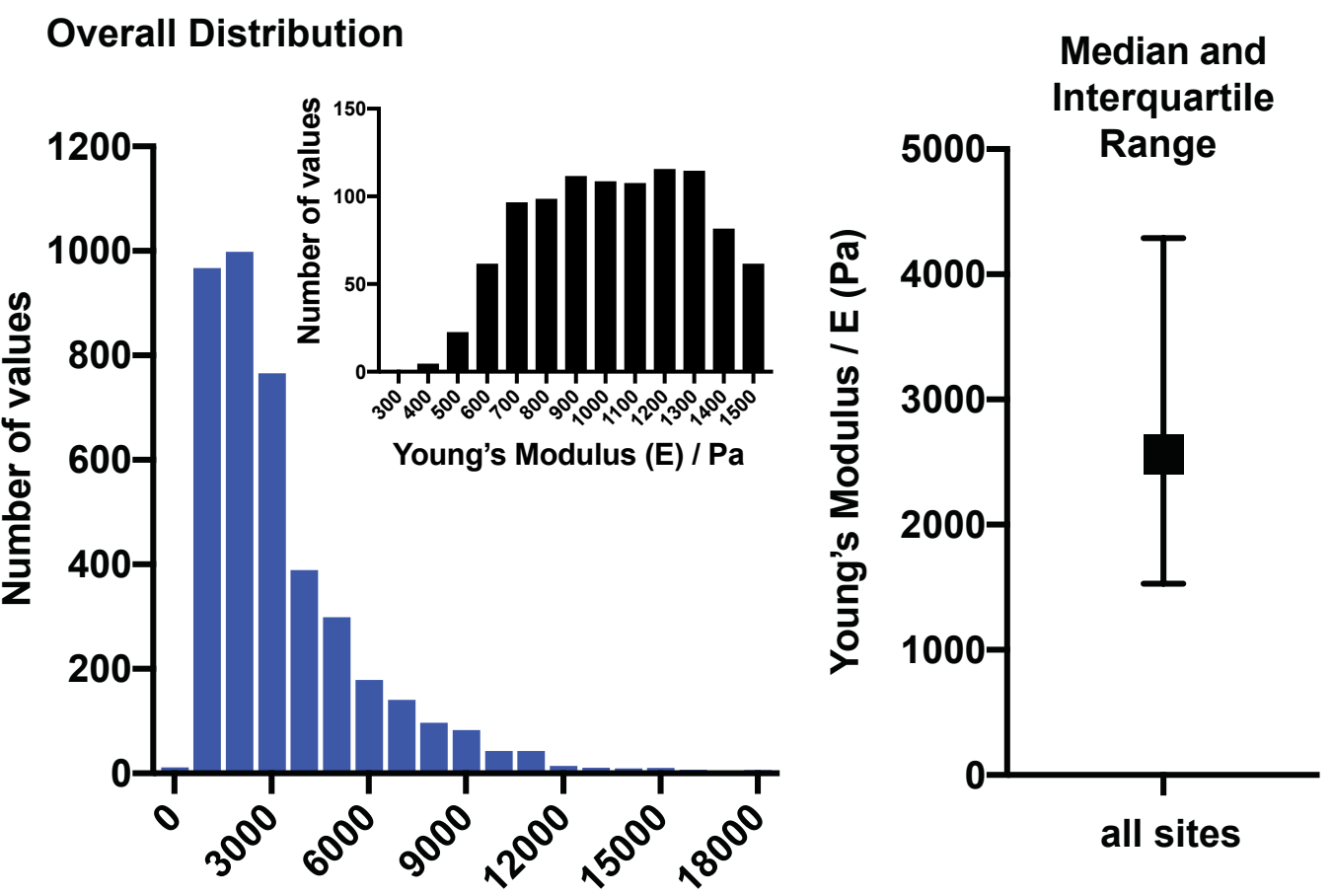

Young's Modulus (E) / Pa

Figure 2: (Left panel) A histogram representing the overall distribution of Young's moduli (E)/Pa across all probed ovaries (within culture media, $n=4$ ovaries). $X$-axis values correspond to upper limit of bin, e.g. $3000 \mathrm{~Pa}$ implies values between 2000 and $3000 \mathrm{~Pa}$. Each value within the histogram represents one force curved produced. Inset shows the low-stiffness part of the histogram at higher resolution (smaller bin size). (Right panel) Median and interquartile range of the data across all samples.

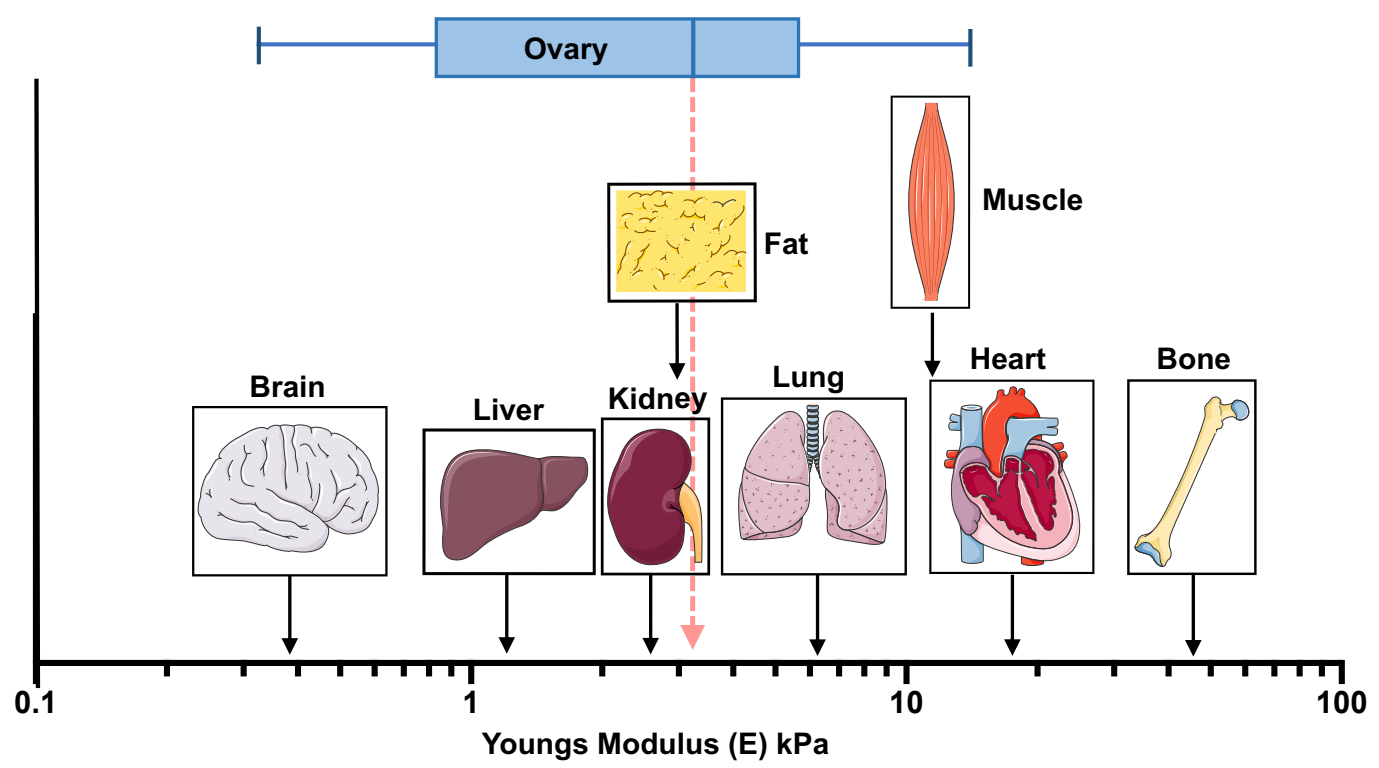

Figure 3: The measured stiffness of the ovary placed in the context of other mouse tissues (data for other tissues reproduced and figure adapted from (66)). 
The ovary is softest at the centre and edge, with an intermediate zone of maximal stiffness

Line profiles of stiffness across the ovaries were plotted (Figure 4). The raw data for a representative example (Figure 4A) shows how the softer ovary sits within the stiffer agar gel used as a matrix. We then extract the ovary data alone (representative example Figure B), displaying the mean and standard deviation of Young's modulus values found within the local stiffness map at a specific location (X in the schematic Figure 1D). The experiment was repeated across a total of seven ovaries from different animals (Figure 4B, 4C). A characteristic double-peak structure was identified and we now discuss this.

The edges of the ovary are softer, with a Young's Modulus that varies between ovaries but is in the region of $2-3 \mathrm{kPa}$. Moving towards the centre of the ovary, the stiffness rises to a peak which again varies but is significantly greater, with an average peak value of $\sim 7 \mathrm{kPa}$ (Figure 4B). This peak stiffness does not occur at the centre of the ovary, rather twin peaks are observed on either side of the centre, which itself is soft, similar in stiffness to the edge region. This double-peak structure of the stiffness linescan profile is observed in 6 out of 7 ovaries (Figure 4C). Look in particular at the averaged line-scan (Figure 4D, created by taking the mean of the ovary profiles). Hence, we can give a well-defined description of the microenvironment that will be experienced by follicles throughout the ovary.

It is interesting to note that the observed spatial variation of stiffness within the ovary had not been previously predicted, emphasizing the importance of directly mapping tissue stiffness using approaches such as AFM. 


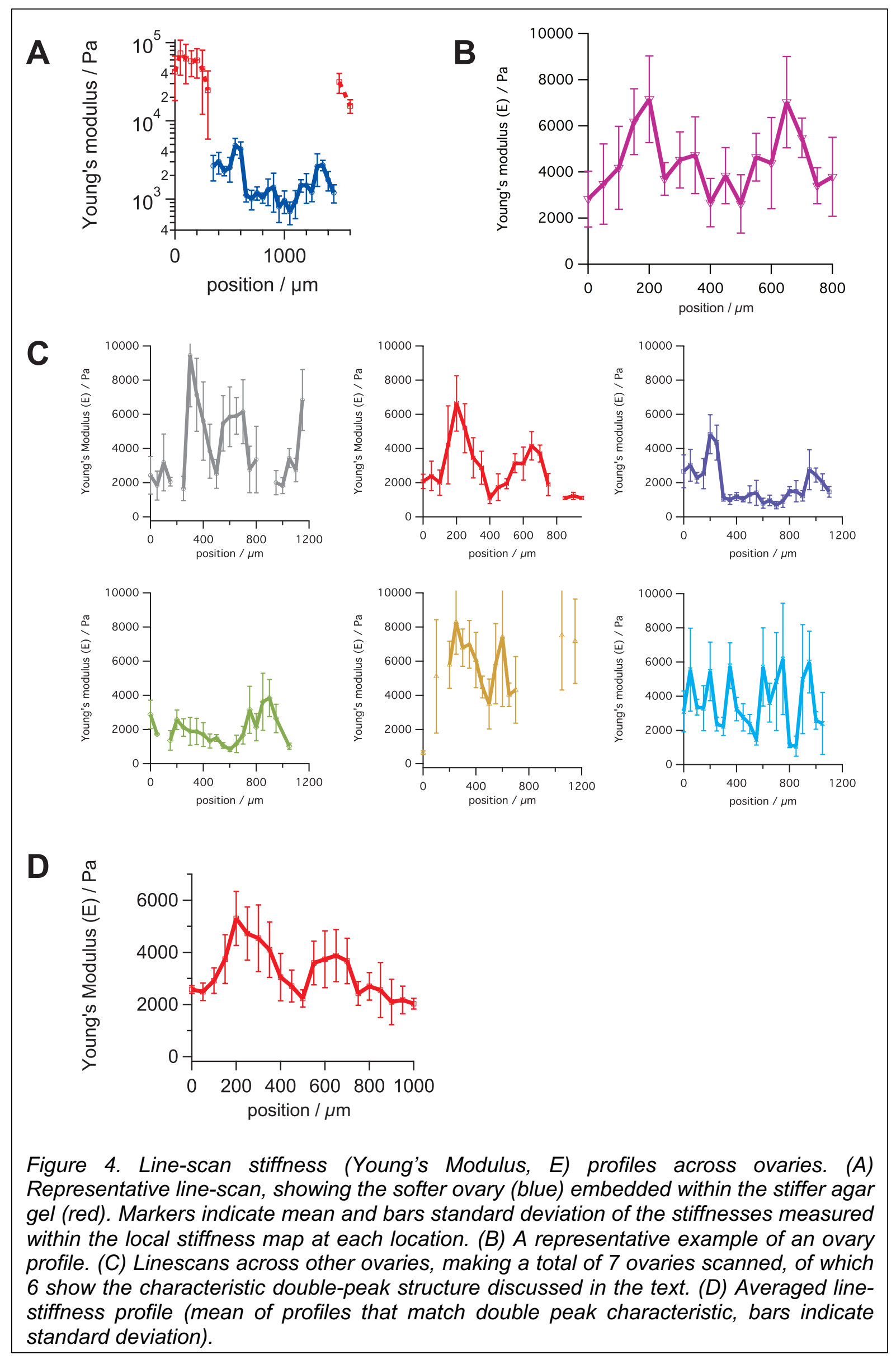


Higher-stiffness regions in the ovary are associated with larger follicles, and not with inter-follicular $E C M$

Stiffness in tissues is often associated with ECM components, notably collagens. A common model is that collagens are primarily responsible for the stiffness of tissues with a high collagen concentration typically thought to correspond to a stiff microenvironment and vice versa (44). Indeed this concept has been applied to the ovary by several authors who have postulated that the high concentration of collagen often seen at the edge [cortex] of the ovary should make it the stiffest region of the ovarian microenvironment $(24,45)$. However we have seen clearly from our AFM measurements that directly characterize mechanics that the edge of the ovary is not the stiffest region. Hence we now examine quantitatively whether the stiffer regions of the ovary indeed correspond to the highest collagen concentration, or whether other mechanisms are at work.

Following AFM analysis, the probed bisected ovary was fixed in formalin, paraffin embedded and sectioned. Collagen IV, which is highly expressed in the basal lamina and theca layer (46) was localized in the probed section (Figure 1A, representative image). To generate a clear idea of the quantity of collagen IV in the different parts of the ovary, used as a marker for the inter-follicular space, we determined the average amount of collagen IV per unit area as a function of distance from the ovarian center. Mathematically, we calculated the two-dimensional radial distribution function $g(r)$ of the collagen IV immunofluorescence intensity, defined as the intensity per unit area in an annulus [ring] whose outer edge is distance $r$ from the ovary center (Figure 5A). The results are consistent across ovaries (Figures 5B,C). There is a region of higher collagen IV high at lower values of $r$, near the ovary center. Another region of higher collagen IV density is seen near the ovary edge. In between, there is a region of lower collagen IV density.

It is hence immediately clear that the regions of high collagen IV density do not correspond to the regions of high stiffness identified above. Rather there is an inverse relationship. The regions of higher collagen IV density at the center and edge are also regions of lower Young's modulus, while the region of lower collagen IV density in between the two corresponds to a peak in Young's modulus. 


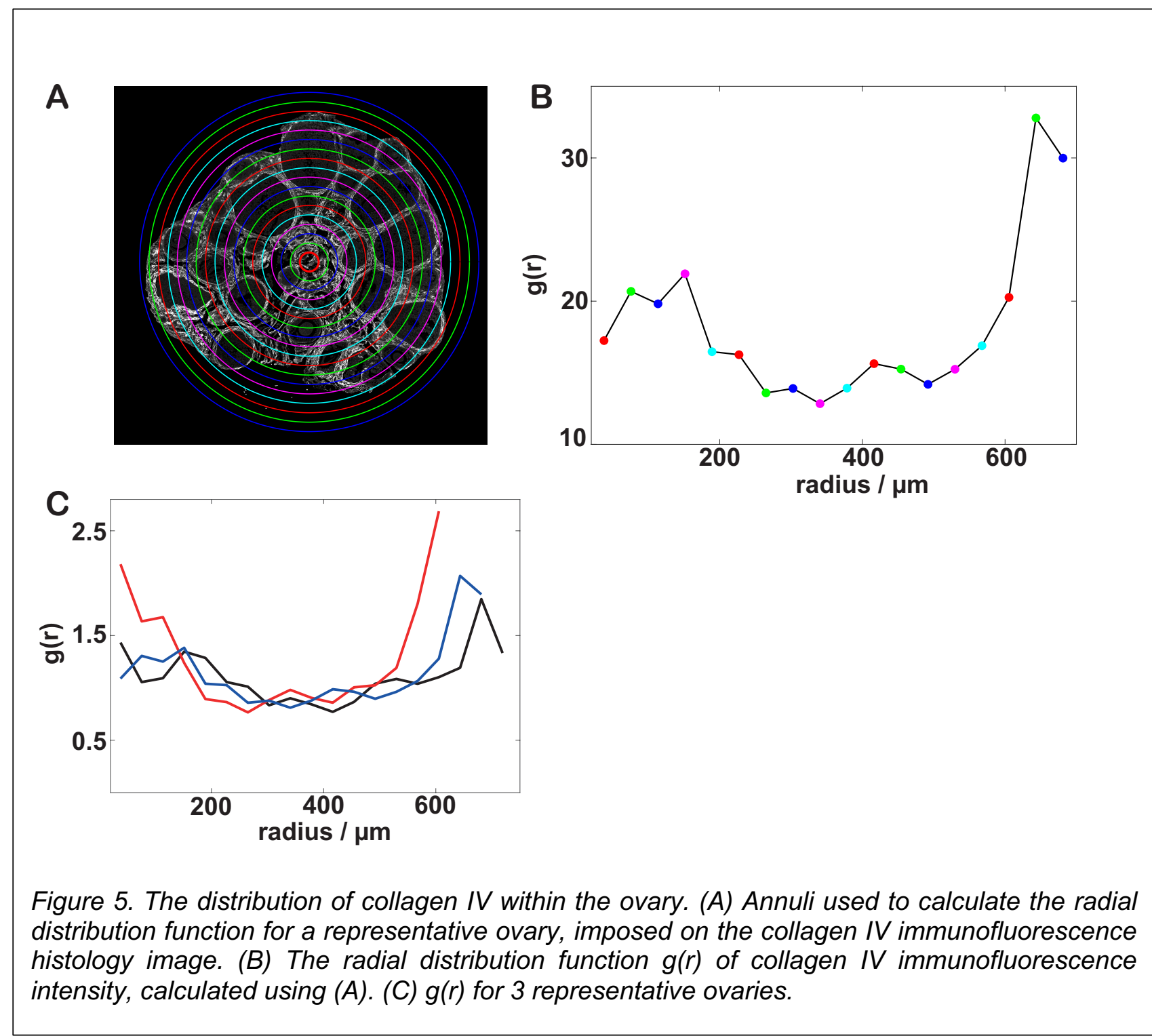

Looking in detail at the immunofluorescence images, we see why the overall collagen IV density is low in the high-stiffness region: this area is dominated by larger follicles, with the follicular interior consisting of granulosa cells which express little or no collagen IV, the oocyte and, in some larger follicles, a fluid-filled antrum (a cavity formed in the granulosa cell layer). For the specific case of the ovary, therefore, it seems that the mechanical properties of these large follicles, rather than of the extra-follicular ECM, is the dominant factor in determining which areas of the ovary exhibit high vs low stiffness. Note that these results apply to reproductively young ovaries as measured here: it is possible that age-induced fibrosis may develop a greater role for inter-follicle collagens as the ovary ages (31). 
While the dominance of follicles as drivers of high stiffness is clear from our results, the microscopic origin of this stiffness may be attributable to a range of different components that make up the follicle. The oocyte itself has been measured to have a high stiffness (47), although this may depend strongly on developmental stage. Equally, the follicle itself contains some collagen I (20). F-actin is likely to participate, given the observation of highly-defined rings of F-actin rich cells surrounding the oocyte and in the theca layer (4). Finally, follicles as growing tissues, will intrinsically build up an internal pressure (48) rendering them prestressed and difficult to compress further.

\section{High resolution measurements show mechanical microstructuring of the ovary on a local scale}

Having considered how the mechanical environment varies on a large scale across the ovary, we now consider local variations. Recall that, at each location within the ovary a $30 \times 30 \mu \mathrm{m}$ grid of measurements was mapped with up to 36 indentations, we now proceed to consider the variation within the maps themselves (rather than simply the map average plotted in Figure 4). Taking a simple approach, we can see comparing different locations that the standard deviation of Young's Modulus as a fraction of the mean for each location remains roughly constant at roughly one third across many locations and several ovaries (Figure 6B). That is, the stiffest locations showed the most variation. Plotting selected local stiffness maps (Figure 6A): it is clear that the locations of greatest stiffness (the peaks on the trans-ovary profiles (Figure 4)), exhibit very substantial variation. For example in the peak-located heat map 6Aii we see a full stiffness range of almost $8 \mathrm{kPa}$. In comparison to the gap between mean stiffnesses at peak and trough, which is roughly 4-5 $\mathrm{kPa}$ (see above), the local variation is hence smaller but still very significant. Looking at the local stiffness maps themselves, we can see that higher and lower Young's Modulus values are not randomly distributed within the maps but rather there are clear spatial correlations, i.e. there are clear regions of higher and localized stiffness within the maps. This indicates the presence of mechanical microstructure, i.e. regions of high and low stiffness can be discerned on the $\sim 10 \mu \mathrm{m}$ lengthscale.

In contrast to the large-scale variations in stiffness that we observe across the ovary (Figure 2), this local microstructure is clearly not due to the presence or absence of larger follicles, since these are 
typically $\sim 100 \mu \mathrm{m}$ or more across. Rather these variations must represent microstructural variations within the ECM, between ECM or cellular components in the follicle.

\section{Ovarian profile locations / Young's Modulus (Pa)}

A.

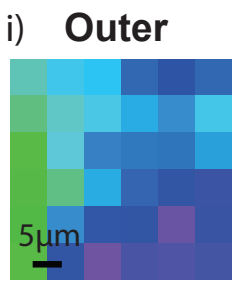

ii) Peak

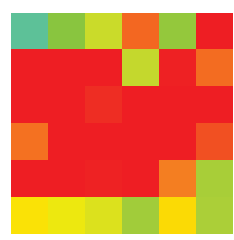

B.

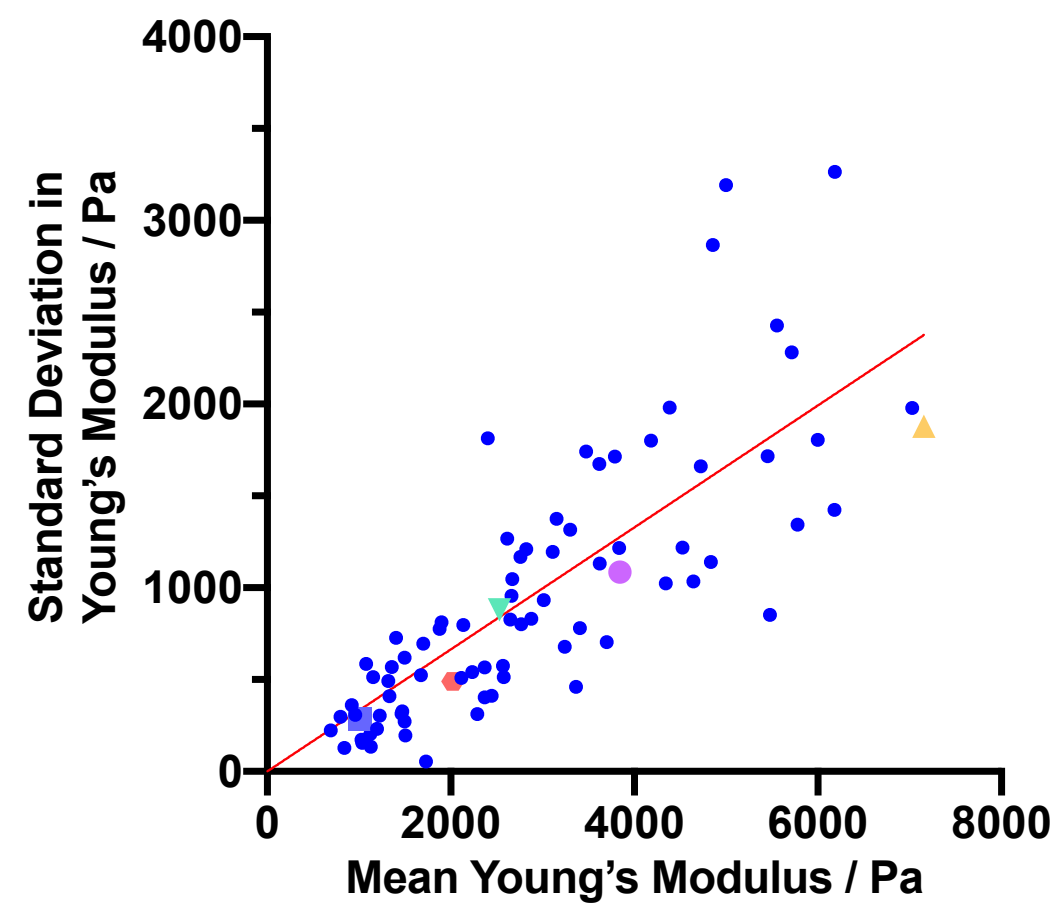

iii) Centre

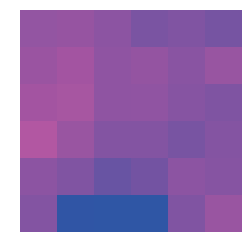

iv) Peak
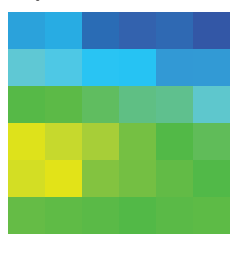

O

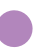

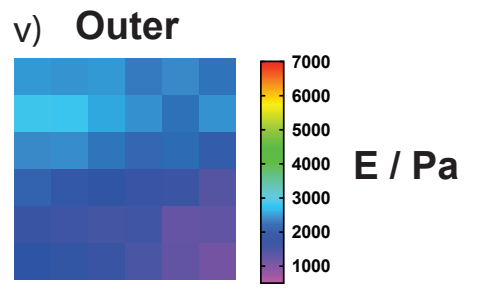

Point below 
characterized by a single Young's modulus. While unsurprising, it is intriguing to consider that in real tissue, microstructural variations in mechanics may be very substantial. We do not know how tissues respond to mechanically microstructured materials and how this may differ from their responses in the homogeneous case.

\section{Conclusion}

We have measured for the first time, and mapped spatially, the mechanical properties of the mouse ovary interior. The Young's Modulus ranges between approximately $0.5-10 \mathrm{kPa}$, with substantial spatial variation on a microstructural level, in addition to larger-scale differences between the different regions of the ovary. This complex structuring emphasizes that tissue components such as cells and follicles experience a complex mechanical microenvironment that cannot be reduced to a single number such as a tissue-scale Young's modulus. Analyzing largescale spatial variations across the ovary shows lower stiffness regions at the ovarian edge and centre, with regions of peak stiffness in between. The areas of low stiffness coincide with a high density of collagen IV, contradicting previous assumptions that the ovarian microenvironment is mechanically dominated by extra-follicular ECM. Rather the higher stiffness regions are dominated by large developmentally advanced follicles, suggesting that these structures should be considered mechanically dominant. The mechanical importance of these follicles stands in contrast to the widespread reductive assumption that stiffer regions can be identified by considering only one component such as collagens. The mechanical characterization of the ovary will be pivotal in understanding how mechanical effects impact follicle development and the development of diseases including PCOS and POI. Equally, a knowledge of the real mechanical properties of the ovarian microenvironment will guide the design of ex-vivo tissue-engineered mimic ovaries. 


\section{Materials and Methods}

\section{Ovary Collection}

Whole ovaries were collected from female C57BL/6 mice pups (Charles River) euthanased at days 25-29 post-partum. Mice were housed in accordance with the Animals (Scientific Procedures) Act of 1986 and associated Codes of Practice.

\section{Atomic Force Microscope (AFM) indentation measurements}

The ovaries were removed and cleaned of extraneous tissue (uterus, oviduct, fat) using insulin needles over a heated microscope stage $\left(37^{\circ} \mathrm{C}\right)$ in $\mathrm{L}-15$ isolation media (Life Technologies, Paisley, United Kingdom) supplemented with 1\% (weight/volume) bovine serum albumin (Sigma). A $2 \%$ agarose (Sigma) solution was created and allowed to cool before being placed into a glass cavity block $(40 \mathrm{~mm} \times 40 \mathrm{~mm})$. The ovary was immersed and positioned within the agarose and allowed to solidify completely. The agarose platform was then dissected through the centre of the ovary using a microtome blade (MX-35, Thermo-Fisher) and glued (Loctite) to the base of the heated bio-cell.
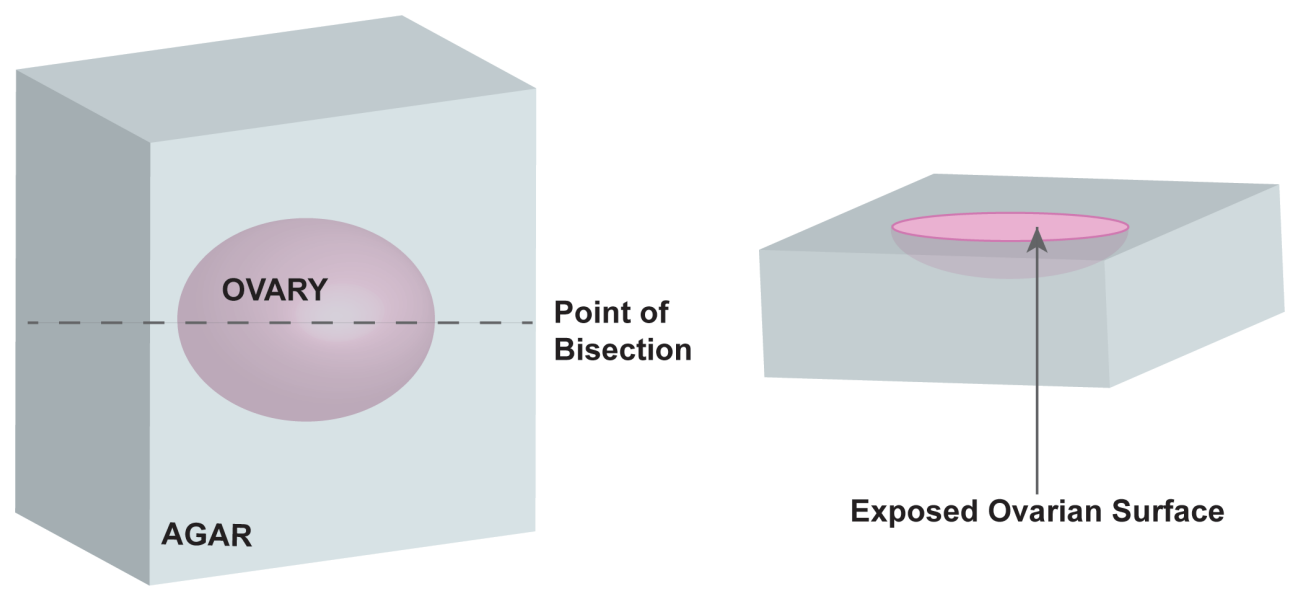

Figure 7: Representation of the technique required to prepare the sample before measurement. The ovary is immersed within a $2 \%$ agar solution and bisected, exposing its interior for analysis, prior to being placed within the AFM.

An atomic force microscope (Asylum MFP-3D, Oxford Instruments) mounted on an incorporated inverted light microscope was used to measure material properties by indentation across a 
transverse section of the ovary. The colloidal probe used for all indentations had a spring constant, $k$, of between $0.02-0.77 \mathrm{~N} / \mathrm{m}$ with a $10.8 \mu \mathrm{m}$ polystyrene sphere attached to the tip of the probe (sQUBE, Bickenbach, Germany). A fluid cell was used to maintain samples within culture medium or water at a constant $37^{\circ} \mathrm{C}$. Before each experiment the inverse optical lever sensitivity (Invols) in water was calibrated by collecting a force curve on a hard surface (glass). The spring constant was determined on the thermal noise spectrum using the instrument manufacturer's software.

Samples were equilibrated on the heated AFM stage at $37^{\circ} \mathrm{C}$, under $\mathrm{L}-15$ cell culture medium or deionized water (MilliQ), before data collection occurred. Using the microscope viewing display, the probe was lowered over a region of agarose close to but outside the tissue itself. Using the built in micrometer-driven sample stage, indentation-based mapping of mechanical properties was then carried out at a series of locations, $50-100 \mu \mathrm{m}$ apart along a diameter across the cut exposed ovary surface. At each of these locations, indentation maps were created, consisting of 36 measurements arranged in a $30 \times 30 \mu \mathrm{m}$ square grid with $5 \mu \mathrm{m}$ spacing between measurement sites, with movement within the grid carried out using the AFM piezoelectric drive. To determine repeatability, 3 indentation maps were identically measured at each location.

\section{AFM data analysis and parameter extraction}

The measured indentation curves were analysed to extract the value of Young's modulus. Approaching curves only were analysed, and a custom-built software programme was created in Igor Pro (Wavemetrics Inc.) to treat the data as follows. Initially, each force-extension curve was accepted or rejected based on requiring a minimum of $10 \mu \mathrm{m}$ of overall travel, to exclude curves where the out-of-contact baseline was insufficient. A second criterion of requiring a minimum of $300 \mathrm{nM}$ of cantilever deflection was added as the project progressed. The user was also given the chance to manually reject the few curves where, for example, the out-of-contact baseline was curved due to varying drift. To remove the effect of typically small linear drifts, a linear baseline fitted to the out-of-contact region was first subtracted before the force-extension curves were converted into force-deflection curves using the measured value of the cantilever spring constant. The Young's modulus was determined by a Hertz model fit, made in the region of moderately deep indentation 
(Figure 1E). This was done to exclude the region of initial contact where the apparent stiffness might be affected by surface damage due to the microtome blade. In addition, measuring at a reasonably deep indentation means that the measured Young's modulus is in effect integrated over a larger volume of the sample, implying greater reproducibility. The equation used for fitting was

$$
F=A\left(\delta-\delta_{0}\right)^{3 / 2}
$$

where $F$ is the force, $\left(\delta-\delta_{0}\right)$ the indentation with $\delta_{0}$ being the surface position, and $A$ a prefactor given by (37).

$$
A=\frac{4 E R^{1 / 2}}{3\left(1-\nu^{2}\right)}
$$

The free parameters $A$ and $\delta_{0}$ were determined by least squares fitting (Igor Pro intrinsic function) and then $E$ was determined using the known value of $R$ and an assumed Poisson's ratio of 0.45 , representative of the values quoted for soft tissues $(48,49)$.

The repeatability of the measurements was confirmed by comparing the multiple maps performed at each site. No significant difference was seen between the statistical distribution of Young's Moduli first and second mappings at a given location (Supplementary Figure $1 \mathrm{~A}$ ) and comparing the mappings shows an essentially unchanged picture also when taking spatial location into account (Supplementary Figure 1B). This confirms reproducibility and shows that indentation does not appreciably damage the tissue surface. Once this had been confirmed, first mappings (ie. The first indentation at each site) were used throughout except in some cases where the number of rejections was too high to deliver sufficient good force curves from separate sites in each map, in which case values from first and subsequent mappings were combined to satisfy this criterion.

\section{Histology}

Upon completion of experiments the tissues were fixed in Formalin (10\% Neutral Buffered) (HT5014, Sigma-Aldrich) overnight. Using a microscope stage (Nikon), bisected ovaries were arranged so that the probed surface faced downwards and set in $2 \%$ agar (Sigma). Samples were processed through graded alcohol $(70 \% 1 \mathrm{~h}), 90 \%(1 \mathrm{~h}), 100 \%(3 \times 1 \mathrm{~h}))$ and then placed in Histoclear (Cat. No HS-200, National Diagnostics) overnight, followed by a further $1 \mathrm{~h}$ the following morning. Samples were then 
embedded in paraffin wax for 2 hours at $55^{\circ} \mathrm{C}$, at the correct orientation so that the probed ovarian surface could be analysed.

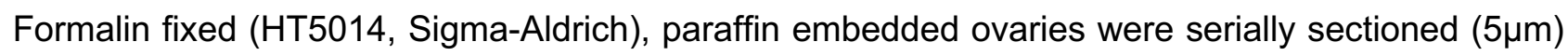
parallel with the cut surface using a Leica RM2135 microtome (Leica Microsystems UK Ltd. Milton Keynes UK) and mounted onto slides (SuperFrost Plus, VWR International Ltd.)

\section{Haematoxylin and Eosin Staining}

Samples were sectioned and stained using haematoxylin and eosin (H\&E), following the standard protocol. Briefly, the tissue sections were dewaxed using xylene (Scientific Laboratory Supplies) and hydrated by passing through decreasing concentration of alcohol (100\%, 90\%, 70\%). Following staining using Gills haematoxylin () for 3 minutes and washed under running tap water. The sections were then differentiated by submersion in $1 \%$ acid alcohol (1\% $\mathrm{HCL}$ and $70 \%$ alcohol) and again washed in water. 1\% Eosin was applied for 5 seconds followed by dehydrated in increasing concentration of alcohol and cleared in xylene before mounting with mounting media (Eukitt, Scientific Laboratory Supplies).

\section{Immunofluorescence imaging}

Slides with central sections of ovary were de-waxed in Histoclear (National Diagnostics) for 5 mins $x$, then re-hydrated in $100 \%, 95 \%$ and $70 \%$ ethanol. They were finally washed in deionised water for $2 \times 5$ mins. Slides were boiled for 20 mins in a citrate buffer for antigen retrieval and left to cool for 30 mins. Slides were consequently washed in phosphate buffered saline (PBS) for $3 \times 10$ mins. To reduce non-specific binding, slides were blocked with $5 \%$ normal goat serum (heat-inactivated) or $10 \%$ normal donkey serum in PBS, supplemented with $4 \%$ BSA () for 30 mins at RT. Primary antibodies were diluted in either $5 \%$ or $1 \%$ serum/BSA in PBS and applied to the sections overnight at $4^{\circ} \mathrm{C}$.

The following day, slides were washed in PBS $3 \times 10$ mins. A secondary antibody conjugated to Alexa-Fluor ${ }^{\mathrm{TM}} 488$ (Abcam, A150077) was added to all slides, for $1 \mathrm{~h}$ at RT, in the dark. After, slides were rinsed in PBS for $3 \times 5$ mins and stained with $1 \% \mathrm{~g} / \mathrm{mL}$ 4,6-diamino-2-phenylindole (DAPI - 
Molecular Probes) for 3 mins in the dark at RT. Slides were mounted in 'Prolong Gold' Antifade P36931 (Molecular Probes) containing DAPI and left at RT for $24 \mathrm{~h}$ before imaging. The slides were then imaged using a Leica inverted SP5 confocal laser-scanning microscope (Leica Microsystems, Wetzlar, Germany). Individual images were taken across the entire sample to generate a full ovarian cross-sectional image. In the ovaries selected for quantitative analysis, laser and detector settings were held constant across all samples and positions, and these ovaries were also stained simultaneously.

\section{Image analysis}

Individual images were stitched together in two distinct ways. For pure visualization a manuallybased stitching software DoubleTake (Echo One, Denmark) was used across all channels. For image quantification, scans were stitched together in FIJI using the function pairwise stitching. These images were segmented in MATLAB (The MathWorks, Natick, MA) using a graph cut segmentation, with morphological closing subsequently applied to smooth edges (MATLAB function imclose, disc radius 12 pixels). The centre of each ovarian section was calculated from the segmentation.

To quantify the distribution of collagen IV within the ovary, a radial distribution function was calculated, measuring the average intensity of collagen fluorescence in annular regions of width $\Delta r$ centred on each ovarian section. Specifically, for $r_{i}=i \Delta r$

$$
g\left(r_{i}\right)=\frac{1}{A_{i}} \sum_{\Omega_{\mathrm{i}}} I\left(x_{j}, y_{j}\right), \quad \Omega_{i}=\left\{\left(x_{j}, y_{j}\right) \mid\left(x_{j}, y_{j}\right) \in A_{i}\right\}
$$

Where $I(x, y)$ is the intensity and $A_{i}$ is the area of the annulus $r_{i}-\Delta r<r<r_{i}$ lying within the ovarian section thus adjusting for edge effects. Of course, where the annulus lies entirely within the tissue section $A_{i}=\pi\left(r_{i}^{2}-\left(r_{i}-\Delta r\right)^{2}\right)$. The annulus width $\Delta r=37.8 \mu \mathrm{m}$ throughout.

Acknowledgements: We acknowledge funding from the EPSRC (Doctoral Training Programme scholarship to TH). Additional consumable funds were kindly provided by the Genesis Research Trust (GRT). 
bioRxiv preprint doi: https://doi.org/10.1101/2021.01.03.425098; this version posted January 4,2021 . The copyright holder for this preprint (which was not certified by peer review) is the author/funder, who has granted bioRxiv a license to display the preprint in perpetuity. It is made available under aCC-BY-NC-ND 4.0 International license.

\section{References}

1. Comim F V., Hardy K, Robinson J, Franks S. Disorders of follicle development and steroidogenesis in ovaries of androgenised foetal sheep. J Endocrinol. 2015 Apr;225(1):39_ 46.

2. Franks S, Stark J, Hardy K. Follicle dynamics and anovulation in polycystic ovary syndrome. Hum Reprod Update. 2008;14(4):367-78.

3. Knight PG, Glister C. TGF- $\beta$ superfamily members and ovarian follicle development. Reproduction. 2006;132(2):191-206.

4. Da Silva-Buttkus P, Jayasooriya GS, Mora JM, Mobberley M, Ryder TA, Baithun M, et al. Effect of cell shape and packing density on granulosa cell proliferation and formation of multiple layers during early follicle development in the ovary. J Cell Sci. 2008 Dec 1;121(23):3890-900.

5. Drummond AE. The role of steroids in follicular growth. Reprod Biol Endocrinol. $2006 \mathrm{Apr}$ 10;4:16.

6. Gill A, Jamnongjit M, Hammes SR. Androgens Promote Maturation and Signaling in Mouse Oocytes Independent of Transcription: A Release of Inhibition Model for Mammalian Oocyte Meiosis. Mol Endocrinol. 2004;18(1):97-104.

7. Stubbs SA, Webber LJ, Stark J, Rice S, Margara R, Lavery S, et al. Role of insulin-like growth factors in initiation of follicle growth in normal and polycystic human ovaries. J Clin Endocrinol Metab. 2013;98(8):3298-305.

8. Kawamura K, Cheng Y, Suzuki N, Deguchi M, Sato Y, Takae S, et al. Hippo signaling disruption and Akt stimulation of ovarian follicles for infertility treatment. Proc Natl Acad Sci U S A. 2013;110(43):17474-9.

9. Cheng Y, Feng Y, Jansson L, Sato Y, Deguchi M, Kawamura K, et al. Actin polymerizationenhancing drugs promote ovarian follicle growth mediated by the Hippo signaling effector YAP. FASEB J. 2015;29(6):2423-30.

10. Carracedo A, Pandolfi PP. The PTEN-PI3K pathway: Of feedbacks and cross-talks. Oncogene. 2008;27(41):5527-41.

11. Jagarlamudi K, Liu L, Adhikari D, Reddy P, Idahl A, Ottander U, et al. Oocyte-specific deletion of Pten in mice reveals a stage-specific function of PTEN/PI3K signaling in oocytes in controlling follicular activation. PLoS One. 2009;4(7):e6186.

12. Reddy P, Liu L, Adhikari D, Jagarlamudi K, Rajareddy S, Shen Y, et al. Oocyte-specific deletion of pten causes premature activation of the primordial follicle pool. Science (80- ). 2008;319(5863):611-3.

13. Cecconi $S$, Mauro A, Cellini V, Patacchiola $F$. The role of Akt signalling in the mammalian ovary. Int J Dev Biol. 2012;56(10-12):809-17.

14. West ER, Xu M, Woodruff TK, Shea LD. Physical properties of alginate hydrogels and their effects on in vitro follicle development. Biomaterials. 2007;28(30):4439-48.

15. Choi JK, Agarwal $\mathrm{P}$, Huang $\mathrm{H}$, Zhao $\mathrm{S}, \mathrm{He} X$. The crucial role of mechanical heterogeneity in regulating follicle development and ovulation with engineered ovarian microtissue. Biomaterials. 2014;35(19):5122-8.

16. Hornick JE, Duncan FE, Shea LD, Woodruff TK. Isolated primate primordial follicles require a rigid physical environment to survive and grow in vitro. Hum Reprod. 2012;27(6):1801-10.

17. Desai N, Abdelhafez F, Calabro A, Falcone T. Three dimensional culture of fresh and vitrified mouse pre-antral follicles in a hyaluronan-based hydrogel: A preliminary investigation of a novel biomaterial for in vitro follicle maturation. Reprod Biol Endocrinol. 2012;10(1):29.

18. Laronda MM, Rutz AL, Xiao S, Whelan KA, Duncan FE, Roth EW, et al. A bioprosthetic ovary created using $3 \mathrm{D}$ printed microporous scaffolds restores ovarian function in sterilized mice. Nat Commun [Internet]. 2017;8(May):1-10. Available from: http://dx.doi.org/10.1038/ncomms15261

19. Shikanov A, Xu M, Woodruff TK, Shea LD. Interpenetrating fibrin-alginate matrices for in vitro ovarian follicle development. Biomaterials. 2009;30(29):5476-85.

20. Berkholtz CB, Lai BE, Woodruff TK, Shea LD. Distribution of extracellular matrix proteins type I collagen, type IV collagen, fibronectin, and laminin in mouse folliculogenesis. Histochem Cell Biol. 2006;126(5):583-92. 
bioRxiv preprint doi: https://doi.org/10.1101/2021.01.03.425098; this version posted January 4,2021 . The copyright holder for this preprint (which was not certified by peer review) is the author/funder, who has granted bioRxiv a license to display the preprint in perpetuity. It is made available under aCC-BY-NC-ND 4.0 International license.

21. Lind AK, Weijdegård B, Dahm-Kähler P, Mölne J, Sundfeldt K, Brännström M. Collagens in the human ovary and their changes in the perifollicular stroma during ovulation. Acta Obstet Gynecol Scand. 2006;85(12):1476-84.

22. Rodgers HF, Irvine CM, Van Wezel IL, Lavranos TC, Luck MR, Sado Y, et al. Distribution of the $\alpha 1$ to $\alpha 6$ chains of type IV collagen in bovine follicles. Biol Reprod. 1998;59(6):1334-41.

23. Zhao Y, Luck MR. Gene expression and protein distribution of collagen, fibronectin and laminin in bovine follicles and corpora lutea. J Reprod Fertil. 1995;104(1):115-23.

24. Woodruff TK, Shea LD. A new hypothesis regarding ovarian follicle development: Ovarian rigidity as a regulator of selection and health. J Assist Reprod Genet. 2011;28(1):3-6.

25. Chiti MC, Dolmans MM, Mortiaux L, Zhuge F, Ouni E, Shahri PAK, et al. A novel fibrinbased artificial ovary prototype resembling human ovarian tissue in terms of architecture and rigidity. J Assist Reprod Genet. 2018;35(1):41-8.

26. Agarwal P, Choi JK, Huang H, Zhao S, Dumbleton J, Li J, et al. A Biomimetic Core-Shell Platform for Miniaturized 3D Cell and Tissue Engineering. Part Part Syst Charact. 2015;32(8):809-16.

27. Clarke HG, Hope SA, Byers S, Rodgers RJ. Formation of ovarian follicular fluid may be due to the osmotic potential of large glycosaminoglycans and proteoglycans. Reproduction. 2006;132(1):119-31.

28. Irving-Rodgers HF, Morris S, Collett RA, Peura TT, Davy M, Thompson JG, et al. Phenotypes of the ovarian follicular basal lamina predict developmental competence of oocytes. Hum Reprod. 2009;24(4):936-44.

29. Rodgers RJ, Irving-Rodgers HF. Formation of the ovarian follicular antrum and follicular fluid. Biol Reprod. 2010;82(6):1021-9.

30. Heeren AM, Van Iperen L, Klootwijk DB, De Melo Bernardo A, Roost MS, Fernandes MMG, et al. Development of the follicular basement membrane during human gametogenesis and early folliculogenesis. BMC Dev Biol [Internet]. 2015 Jan 21 [cited 2020 May 12];15(1):4. Available from: http://www.biomedcentral.com/1471-213X/15/4

31. Amargant F, Manuel SL, Tu Q, Parkes WS, Rivas F, Zhou LT, et al. Ovarian stiffness increases with age in the mammalian ovary and depends on collagen and hyaluronan matrices. Aging Cell. 2020;00(e13259).

32. Elkin BS, Azeloglu EU, Costa KD, Morrison B. Mechanical heterogeneity of the rat hippocampus measured by atomic force microscope indentation. J Neurotrauma. 2007;24(5):812-22.

33. Lekka M, Gil D, Pogoda K, Dulińska-Litewka J, Jach R, Gostek J, et al. Cancer cell detection in tissue sections using AFM. Arch Biochem Biophys. 2012;518(2):151-6.

34. Iwashita M, Kataoka N, Toida K, Kosodo Y. Systematic profiling of spatiotemporal tissue and cellular stiffness in the developing brain. Dev. 2014;141(19):3793-8.

35. Christ AF, Franze K, Gautier H, Moshayedi P, Fawcett J, Franklin RJM, et al. Mechanical difference between white and gray matter in the rat cerebellum measured by scanning force microscopy. J Biomech. 2010;43(15):2986-92.

36. Engler AJ, Griffin MA, Sen S, Bönnemann CG, Sweeney HL, Discher DE. Myotubes differentiate optimally on substrates with tissue-like stiffness: Pathological implications for soft or stiff microenvironments. J Cell Biol [Internet]. 2004 [cited 2018 Sep 19];166(6):87787. Available from: http://www.jcb.org/cgi/doi/10.1083/jcb.200405004JCBArticle

37. Harris AR, Charras GT. Experimental validation of atomic force microscopy-based cell elasticity measurements. Nanotechnology. 2011;22(34).

38. Samani A, Zubovits J, Plewes D. Elastic moduli of normal and pathological human breast tissues: An inversion-technique-based investigation of 169 samples. Phys Med Biol. 2007;52(6):1565-76.

39. Sicard D, Fredenburgh LE, Tschumperlin DJ. Measured pulmonary arterial tissue stiffness is highly sensitive to AFM indenter dimensions. J Mech Behav Biomed Mater. 2017;74:11827.

40. Engler AJ, Rehfeldt F, Sen S, Discher DE. Microtissue Elasticity: Measurements by Atomic Force Microscopy and Its Influence on Cell Differentiation. Methods Cell Biol. 2007;83:52145.

41. Swift J, Ivanovska IL, Buxboim A, Harada T, Dingal PCDP, Pinter J, et al. Nuclear lamin-A scales with tissue stiffness and enhances matrix-directed differentiation. Science (80- ). 
2013;341(6149).

42. Jin SY, Lei L, Shikanov A, Shea LD, Woodruff TK. A novel two-step strategy for in vitro culture of early-stage ovarian follicles in the mouse. Fertil Steril. 2010;93(8):2633-9.

43. Ahn J II, Kim GA, Kwon HS, Ahn JY, Hubbell JA, Song YS, et al. Culture of preantral follicles in poly(ethylene) glycol-based, three-dimensional hydrogel: A relationship between swelling ratio and follicular developments. J Tissue Eng Regen Med. 2015;9(3):319-23.

44. Paszek MJ, Zahir N, Johnson KR, Lakins JN, Rozenberg GI, Gefen A, et al. Tensional homeostasis and the malignant phenotype. Cancer Cell. 2005;8(3):241-54.

45. Hughesdon PE. Morphology and morphogenesis of the stein-leventhal ovary and of socalled "Hyperthecosis." Obstet Gynecol Surv. 1982;37(2):59-77.

46. Hardy K, Mora JM, Dunlop C, Carzaniga R, Franks S, Fenwick MA. Nuclear exclusion of SMAD2/3 in granulosa cells is associated with primordial follicle activation in the mouse ovary. J Cell Sci. 2018;131(17).

47. Murayama $\mathrm{Y}$, Constantinou CE, Omata S. Micro-mechanical sensing platform for the characterization of the elastic properties of the ovum via uniaxial measurement. J Biomech. 2004;37(1):67-72.

48. Littlejohns E, Dunlop CM. Mechanotransduction mechanisms in growing spherically structured tissues. New J Phys. 2018;20(4).

49. Glozman T, Azhari H. A method for characterization of tissue elastic properties combining ultrasonic computed tomography with elastography. J Ultrasound Med. 2010;29(3):387-98.

50. Mitomo M, Uenosono S. Gas pressure sintering of $\beta$-silicon nitride. Vol. 26, Journal of Materials Science. 1991. 3940-3944 p. 


\section{SUPPLEMENTARY INFORMATION}

A

\section{First and Second} Contact Analysis

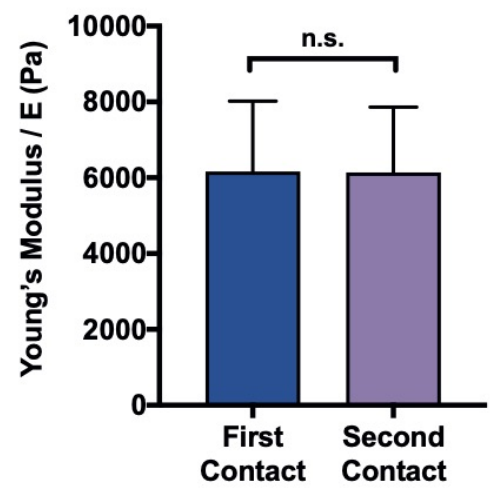

B

1st Contact

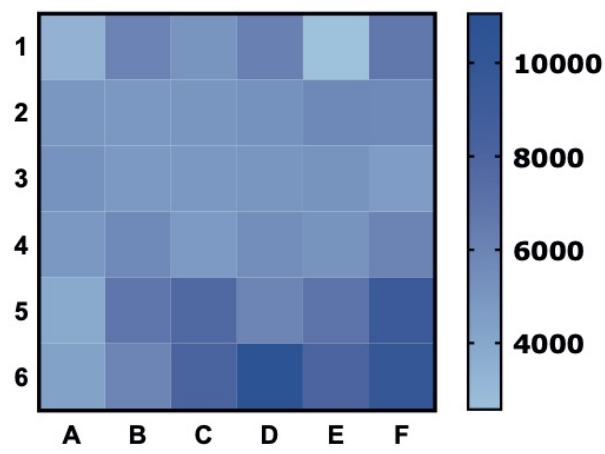

2nd Contact

\section{$\mathrm{E} / \mathrm{Pa}$}

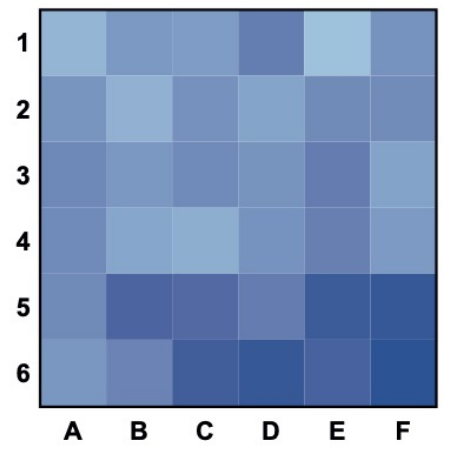

10000

8000

6000

4000

Supplementary Figure 1: First and second compressions of ovaries show no statistically significant difference. A) Graph demonstrating a representation of the difference between first contact and second contact at the same site, there is no significant difference between the two groups demonstrating no change in the results taken from the first or second contact $(P>0.05)$. Normality was assessed using a $D^{\prime}$ Agostino and Pearson test. A paired t test was performed. Graph shows mean and SD B) Heatmaps that show the Young's Modulus at a single position at the first and second contact. 


\section{Literature analysis of permissive versus suppressive controlled-stiffness gels for follicle development}

\begin{tabular}{|l|l|l|l|l|}
\hline Reference & Material & Notes & $\begin{array}{l}\text { G / Pa } \\
\text { (from ref) }\end{array}$ & $\begin{array}{l}\text { E / Pa (our } \\
\text { estimate) }\end{array}$ \\
\hline $\begin{array}{l}\text { West et al. } \\
\text { Ref (14) }\end{array}$ & $\begin{array}{l}\text { Alginate gel } \\
0.7 \%\end{array}$ & $\begin{array}{l}\text { Permissive for growth, } \\
\text { antrum formation and } \\
\text { theca layer formation }\end{array}$ & 203 & 589 \\
\hline $\begin{array}{l}\text { Shikanov et } \\
\text { al. Ref (19) }\end{array}$ & $\begin{array}{l}\text { Fibrin- } \\
\text { Alginate }\end{array}$ & $\begin{array}{l}\text { Stated as approx. upper } \\
\text { limit of permissiveness }\end{array}$ & 300 & 870 \\
\hline $\begin{array}{l}\text { Choi et al., } \\
\text { Ref (15) }\end{array}$ & $\begin{array}{l}\text { Collagen gel } \\
0.5 \%\end{array}$ & $\begin{array}{l}\text { Permissive for follicle } \\
\text { development }\end{array}$ & 116 \\
\hline
\end{tabular}

Supplementary Table 1: Literature analysis showing the stiffness of hydrogels defined as permissive for follicle development by various authors. Values quoted by these authors are shear modulus $G$ (or alternatively storage modulus G'), converted to Young's Modulus using $E=$ $2 G(1+v)(50)$ taking the Poisson's ratio, $v$, as $\sim 0.45$ (49).

\begin{tabular}{|c|c|c|c|}
\hline Primary Antibody & Block Used & $\begin{array}{c}\text { Primary Antibody } \\
\text { Dilution }\end{array}$ & $\begin{array}{c}\text { Secondary Antibody } \\
\text { Dilution }\end{array}$ \\
\hline $\begin{array}{c}\text { Collagen IV } \\
\text { (Millipore \#AB756P) }\end{array}$ & $\begin{array}{c}\text { Normal Goat } \\
\text { Serum (5\%) }\end{array}$ & $\begin{array}{c}1: 100 \text { in (5\% } \\
\text { Serum in PBS) }\end{array}$ & $\begin{array}{c}1: 200 \text { Alexa-Fluor Goat } \\
\text { anti-rabbit 488 (Abcam, } \\
\text { \#A150077) }\end{array}$ \\
\hline
\end{tabular}

Supplementary Table 2: Antibodies used for Immunofluorescence. 